\title{
Éditorial
}

\section{Quelques opinions sur la contention}

"La contention est le plus difficile problème en orthodontie. En fait, c'est LE problème », a dit Oppenheim. Tous les auteurs s'y sont confrontés depuis que l'orthodontie existe. Leurs opinions se croisent ou s'opposent, à la recherche de LA solution.

1728 - FAUCHARD. Fauchard contient les déplacements de dents opérés par luxation. «On aura recours aux lames de plomb qu'on appliquera, l'une sur la surface extérieure des dents, et l'autre sur la surface intérieure, en ayant auparavant garni ces lames avec du linge, ou de la charpie pour empêcher qu'elles glissent sur les dents. On tient ces lames assujetties par le moyen d'un fil enfilé dans une aiguille, que l'on passe dans l'intervalle des dents par les trous de ces mêmes lames, de dehors en dedans et de dedans en dehors, jusqu'à ce que les dents ébranlées soient suffisamment raffermies ». «Enfin on fait une lotion avec quatre onces de vin et une once de miel rosat. »

1857 - SCHANGE. Aucun des nombreux et illustres praticiens du XVIII siècle et de la première moitié du XIX n'a parlé de contention. II faut attendre A. Schange, non pas dans son « Précis sur le redressement des dents » de 1841, mais dans un autre ouvrage, retrouvé par $C$. Demange, dans lequel Schange expose le problème et trouve une solution très honorable. "On aurait tort de croire que, une fois ramenées à la direction normale, tout traitement soit terminé. Il faut maintenir les dents longtemps dans la place qu'on leur a assignée, pour qu'elles y acquièrent la solidité convenable, surtout si la marche qu'on leur a fait faire a été un peu considérable. » En 1857, Schange présente un appareil spécialement conçu pour la contention, le « stéréodonte » (du grec stéréos, solide et odous, odontos, dent). C'est une plaque en or, estampée, recouvrant les bords alvéolaires internes et externes. Les faces occlusales des dents sont dégagées, la plaque est ajustée aux collets. L'appareil se retire facilement, ce qui permet « une hygiène parfaite ».

1880 - KINGSLEY. Kingsley, le grand Kingsley, qui inventa les FEB et le " saut d'occlusion », n'est vraiment pas à l'aise sur la question de la contention. II n'y consacre aucun paragraphe, et c'est dans le cours d'un chapitre de physiologie qu'il en parle un peu. « La tendance des dents à revenir à leur ancienne position a été surestimée »... «L'occlusion des dents est le plus puissant facteur qui assure la stabilité de la nouvelle position.... Le port d'une plaque 
de contention ou de dispositifs fixes est indésirable et discutable. C'est un mal, nécessaire dans quelques cas, mais qu'il faut éviter le plus possible. Si les « irrégularités» sont héréditaires, et si la correction est tardive, il sera plus sûr de maintenir pendant deux ou trois ans. » Mais Kingsley ajoute : “Dans les cas héréditaires traités près de la maturité, nous ne sommes jamais certains que la tendance d'origine des malpositions ne réapparaîtra pas à n'importe quel moment. »

1888 - FARRAR. Farrar consacre 23 pages (sur les 1570 de son livre) au problème de la contention. En voici quelques extraits. "Dans les cas de faible ossification des tissus de support, il peut être impossible de fixer les dents, par n'importe quel moyen. Cela arrive quand des élastiques ou des ligatures ont profondément blessé la gencive, ou ont été perdus et remis plusieurs fois, entraînant un grand nombre de mouvements d'aller et retour, d'où résulte une altération de l'alvéole. " "L'appareil de contention doit être porté de trois semaines à trois ans, ou plus, mais mieux vaut trop que pas assez. En cas de déformations héréditaires, il peut être nécessaire de garder l'appareil de contention toute la vie, mais ces cas ne peuvent être clairement déterminés. »

1907 - ANGLE. Angle rappelle d'abord que : « la contention est trop souvent considérée avec légèreté ». Or, « on y rencontre de plus grands problèmes que pour le traitement ». "La stabilité ne peut être espérée hors d'une parfaite occlusion » (par le jeu des plans inclinés cuspidiens). "Il est improbable que les dents restent en bonne occlusion si une ventilation normale n'est pas rétablie et si la bouche ne reste pas fermée. » «Si la contention n'a concerné qu'une arcade, la récidive surviendra dès la dépose de l'appareil de contention. » "La contention a pour principe de s'opposer au mouvement des dents seulement dans la direction de leur tendance à la récidive. " "L'appareil doit être fixé et scellé. » «Les molaires ne doivent pas être déplacées par version, mais par gression; des dents versées causent une perte de stabilité. »

1908 - CASE. "Les dents déplacées d'une position à une autre sont exposées pendant des années aux forces des tissus environnants qui tendent à les ramener dans la mauvaise position qu'elles occupaient auparavant. Les forces de récidive s'exerceront tant que les fibres ligamentaires, tendues et tordues, n'auront pas retrouvé leur équilibre du fait des changements physiologiques. Le dispositif de contention doit être adapté au mouvement de retour, la direction de celui-ci apparaît en comparant les moulages de début et de fin de traitement. Les dents déplacées lentement peuvent être maintenues moins longtemps. " « Le plus important est une bonne relation cuspidienne, mais il faut dire qu'une bonne occlusion molaire ne garantit pas la stabilité antérieure. » "Les traitements des irrégularités d'origine héréditaires sont parfois impossibles à stabiliser, sauf par un système fixé permanent », ici, Case se réfère à Kingsley. D’autre part, il approuve Angle quand celui-ci souligne l'importance de la ventilation nasale pour la contention du traitement des malocclusions de la classe II, div. 1. Généralement, la contention doit durer au moins deux ans, mais les traitements des patients jeunes peuvent être plus brefs que ceux des patients plus âgés. Les appareils fixes sont supérieurs aux amovibles.

1914 - DEWEY. « La contention, c'est l'application de forces qui maintiennent les dents dans la ligne d'occlusion. » Ces forces sont soit mécaniques, soit naturelles. Les forces mécaniques doivent être passives. Les forces naturelles sont : les plans inclinés cuspidiens, l'harmonie de taille des arcades, des pressions musculaires normales, des points de contact normaux, un métabolisme cellulaire normal, une pression atmosphérique normale (ventilation nasale). 
1919 - HAWLEY. "Il y a douze ou quinze ans, un de mes bons amis, qui s'était confronté aux difficultés de la contention durant plusieurs années, me dit : 'si quelqu'un voulait prendre mes cas quand ils sont finis, les contenir et être responsable d'eux ensuite, je serais heureux de lui donner la moitié des honoraires'. ". Après cette déclaration, Hawley précise que le plus souvent les dents ne retournent pas à leur position d'origine, mais que le résultat, après quelques années, n'est pas satisfaisant. Hawley expose les avantages de la plaque amovible qui désormais porte son nom. Il précise que la plaque doit monter sur les faces linguales jusqu'au bord occlusal, dans la mesure où elle ne gêne pas l'occlusion.

1927 - TACAIL. Tacail, bien que disciple d’Angle, est assez expéditif. « Le mieux est souvent de faire passer de l'état actif à l'état passif le même dispositif. Je recommande, comme contention, un appareil fixe. Baguez les dents qui furent en rotation et réunissez les bagues en les soudant à un fil. Après cette première période, une simple plaque en vulcanite, avec ou sans plan incliné, suffira. »

1928 - QUINTERO. « La contention des résultats acquis est la partie la plus importante du traitement orthodontique, car il importe peu que l'on ait obtenu un succès brillant s'il ne doit être que temporaire. » "L'appareil doit s'opposer à toute tendance à la récidive. Le meilleur des appareils, c'est celui qui a servi au traitement. »

1948 - ANDERSON. "La contention est le plus difficile problème de l'orthodontie. Elle est assurée par des moyens naturels et mécaniques. Les moyens naturels sont:

- le guidage des plans inclinés cuspidiens. La relation de la cuspide mésio-linguale de la première molaire maxillaire dans la fosse centrale de la molaire mandibulaire est plus importante que les relations des cuspides vestibulaires pour la maintenance de l'occlusion;

- les pressions musculaires;

- les contacts et appuis proximaux;

- la relation condylienne;

- la pression atmosphérique (ventilation nasale). »

Anderson recommande de placer sur les canines mandibulaires des bagues réunies par un fil soudé, et au maxillaire une plaque amovible, en vulcanite ou en or, accompagnée d'une lettre au patient le mettant en face de ses responsabilités.

1950 - IZARD. "La contention est cette partie du traitement qui a pour but d'assurer le maintien des résultats obtenus et d'empêcher toute récidive. Elle constitue la seconde période du traitement, aussi importante que la première. » Seule est parfaite la contention par les forces naturelles. C'est-à-dire qu'il faut :

"-supprimer les causes générales et locales des lésions;

- rétablir l'équilibre des forces musculaires, en particulier les forces de mastication et la respiration nasale;

- donner aux arcades une forme et un volume en rapport avec leur base apicale. »

1. La référence exacte de cette fameuse citation était perdue. Madame Filleul l'a retrouvée. Nous la remercions de nous l'avoir communiquée. 
Izard recommande des exercices des muscles élévateurs, orbiculaires et propulseurs (le développement de la force musculaire est à la base de la thérapeutique d'Izard, c'est la « myothérapie » de Rogers).

Il est partisan de la septotomie. La plaque de Hawley doit être portée un temps égal à la durée du traitement actif.

1952 - FISHER. «Quelle que puisse être la complexité de la contention, il y a des facteurs qui peuvent assurer la permanence des résultats du traitement:

- les dents n'ont pas été versées ou déplacées au-delà des limites tolérables de leur os de base;

- la taille et la forme des arcades n'ont pas radicalement changé durant le traitement;

- le nombre des dents déplacées a été réduit au minimum;

- les comportements défavorables ont été corrigés. »

Quand ces conditions sont réunies, on peut se passer d'appareil de contention.

1958 - STRANG. "Chaque cas en malocclusion est naturellement dans un état d'équilibre musculaire qui est fixé, comme le type de croissance. L'opérateur n'a pas à établir un nouvel équilibre des forces, mais à respecter cet équilibre fixe et à y adapter la denture. " « II est important de placer les dents sur l'os de base », comme le dit Tweed. "Peu importe le temps durant lequel sont gardés les dispositifs mécaniques, si l'équilibre musculaire n'est pas respecté et si les dimensions de l'arcade sont changées. » «La distance entre les canines mandibulaires ne peut être augmentée d'une façon stable, que si ces canines sont reculées à la place des premières prémolaires, après l'extraction de celles-ci. »

"La supraclusion récidive dans 25 \% des cas. Trop souvent, la supraclusion est corrigée par une version vestibulaire des incisives. Ces dents devenant plus horizontales, l'excès de recouvrement disparaît. Mais ces incisives vestibulées sont d'autant plus exposées à la pression labiale, et, aussitôt l'appareil enlevé, la supraclusion réapparaît, avec les malpositions incisives. "

"L'occlusion traumatique est la cause la plus fréquente de destruction de l'os alvéolaire. Pour cette raison, l'axe de chaque dent doit correspondre aux forces occlusales exercées. La version distale des molaires pratiquée pour la préparation d'ancrage, doit, ensuite, être éliminée ».

La surcorrection est conseillée. "La durée de la contention et le genre d'appareil ne sont pas le point le plus important. Le talent de l'opérateur à garder les arcades dans l'équilibre des forces est la clé de la stabilité. »

1964 - CHATEAU. « La contention a pour but d'éviter ou de limiter la récidive pendant la période d'équilibration, ou pendant la période où une pathogénie nouvelle ou ancienne persistante risque de reproduire une déformation. C'est dire que les appareils de contention doivent laisser les dents assez libres, afin qu'elles puissent changer d'axe et prendre leur position naturelle d'équilibre. » Une contention nocturne discontinue est généralement préférable, à base d'appareils amovibles passifs. « La facilité ou la difficulté éprouvée à remettre l'appareil après une certaine durée d'abandon est précisément le test, et le seul, qui permet de savoir à quel point l'appareil est nécessaire : on doit le conserver davantage s'il est difficile de le remettre; on peut en espacer le port nocturne s'il revient rapidement et tout à fait à sa place. »

1966 - TWEED. «Tant que les orthodontistes ne sauront pas placer la denture dans une position normale, dans le sens mésio-distal, par rapport aux os de base et des structures de la tête, la contention continuera d'être leur fléau. » « À 
l'époque de l'orthodontie sans extractions, les traitements sans extractions se terminaient par un effondrement de la zone incisive mandibulaire et le retour de la supraclusion. »

Le salut vient du respect des valeurs du triangle facial. Le type de contention dépend du type de croissance :

- croissance de type $A$ (croissance égale au maxillaire et à la mandibule). Plaques de Hawley durant un an, puis fil de canine à canine à la mandibule gardé jusqu'à l'âge de seize ans et plaque nocturne au maxillaire pendant dix-huit mois. Équilibration après six mois de contention ;

- croissance de type $B$ (le maxillaire croît plus vite que la mandibule). Force extra-buccale (Kloen neck gear), portée non pour reculer les dents maxillaires, mais pour freiner la croissance du maxillaire vers l'avant ;

- croissance type C (75 \% des cas. La mandibule croît en bas et en avant plus vite que le maxillaire, et l'angle ANB diminue). Fil de canine à canine à la mandibule et plaque de Hawley au maxillaire.

À la fin du traitement, les molaires maxillaires doivent toujours être inclinées distalement. La cuspide mésio-vestibulaire de la dernière molaire doit être la seule partie de la dent en contact occlusal. Elle est retenue dans cette position par le crochet de la plaque de Hawley.

Il est bon d’ajouter un plan rétro-incisif à la plaque pour empêcher le retour de la supraclusion.

1966 - SALZMANN. Salzmann expose d'abord le rôle des fibres desmodontales, puis il énumère certains facteurs d'instabilité :

- les comportements défavorables ;

- la possibilité d'une double occlusion ;

- la taille de la langue;

- l'expansion des arcades ;

- les mimiques et tics faciaux.

Salzmann examine aussi les facteurs favorables à la stabilité :

- de bonnes relations entre plans inclinés cuspidiens ;

- des arcades bien adaptées l'une à l'autre avec un recouvrement normal ;

- des points de contact de bonne qualité.

Les dents doivent être placées en position d'équilibre statique. Il faut vérifier leur mobilité, celle-ci peut être due à une occlusion traumatique. Il faut veiller au rapport de l'occlusion avec la relation centrée. Une équilibration doit supprimer les contacts prématurés et assurer les mouvements de latéralité et de propulsion.

La surcorrection n'apporte pas d'avantages et peut provoquer une occlusion traumatique.

La contention peut ne durer qu'un an si toutes les dysfonctions ont été corrigées.

1993 - CHATEAU. « Nul n'est sûr de conserver longtemps son alignement impeccable et son occlusion.

Les causes de la récidive sont:

- les mauvaises finitions du traitement », le test condylien montre que les condyles ne sont pas en relation centrée, l'intercuspidie n'est pas parfaite, des prématurités demeurent ; 
- «la persistance d'une ou plusieurs causes », en particulier, le trajet mandibulaire, lors de la mastication, n'est pas symétrique (Planas);

- «le déséquilibre post-orthodontique musculaire, postural, dentaire » (force égressive et mésialante).

CONCLUSION. Le charme de cette confrontation entre dix-sept auteurs vient de sa diversité et de ses contradictions. On y trouvera plus des idées qu'une conduite à tenir. Et la phrase de Hawley est une inépuisable source de méditation...

Opinions rassemblées par Julien Philippe

\section{BIBLIOGRAPHIE}

1. Anderson GM. Practical Orthodontics. Saint-Louis : Mosby, 1948.

2. Angle EH. Treatment of malocclusion of teeth. $7^{\text {th }}$ ed. Philadelphia : SS White Dental Manufactyring Co, 1907.

3. Case C.S. Dental Orthopedia. Chicago : The C.S. Case CY, 1908.

4. Château M. Orthopédie Dento-Faciale. Paris : Masson et Prélat, 1964.

5. Château M. Orthopédie Dento-Faciale. Paris : CdP, 1993.

6. Dewey M. Practical orthodontia. Saint-Louis : Mosby, 1914.

7. Farrar JN. A treatise on the irregularity of the teeth and their correction. Tome I. New-York, 1888.

8. Fauchard P. Le Chirurgien Dentiste ou Traité des Dents. Paris : Mariette, 1728.

9. Fisher B. Orthodontics. Philadelphia: W.B. Saunders, 1952.

10. Hawley CA. A removable appliance. The Dental Cosmos. 1919;LXI(6): 449-455.

11. Izard G. Orthodontie. Paris : Masson, 1950.

12. Kingsley NW. A treatise on oral deformities. London: H.K. Lewis 1880.

13. Quintero JT. Technique orthodontique. Paris : J.B. Baillère, 1928.

14. Salzmann JA. Practice of Orthodontics. Philadelphia : J.B. Lippincott, 1966.

15. Schange JMA. Description du treptodonte et du stéréodonte, appareils nouveaux pour le redressement des dents. Paris : Garnier. 1857.

16. Strang RHW. A textbook of orthodontia. Philadelphia : Lea \& Febiger, 1950.

17. Tacail CP. Notions élémentaires d'Orthodontie. Paris, 1927.

18. Tweed CH. Clinical Orthodontics. Saint Louis : Mosby, 1966.

Les opinions émises n'engagent que leurs auteurs. 